\title{
Strategies for high-performance perovskite solar cells
}

\section{Annie Ng, Zhiwei Ren, Changwen Liu, Aleksandra B. Djurišić, Ruixue Zhu, et al.}

Annie Ng, Zhiwei Ren, Changwen Liu, Aleksandra B. Djurišić, Ruixue Zhu, David Lee Phillips, Charles Surya, "Strategies for high-performance perovskite solar cells," Proc. SPIE 10919, Oxide-based Materials and Devices X, 1091905 (1 March 2019); doi: 10.1117/12.2516757

SPIE. Event: SPIE OPTO, 2019, San Francisco, California, United States 


\title{
Strategies for High Performance Perovskite Solar Cells
}

\author{
Annie $\mathrm{Ng}^{\mathrm{a}}$, Zhiwei Ren ${ }^{\mathrm{b}}$, Changwen Liu ${ }^{\mathrm{b}}$, Aleksandra B. Djurišićc ${ }^{\mathrm{c}}$, Ruixue Zhu ${ }^{\mathrm{d}}$, David Lee \\ Phillips ${ }^{\mathrm{d}}$, and Charles Surya $\mathrm{a}^{\mathrm{e}, *}$ \\ ${ }^{a}$ Department of Electrical and Computer Engineering, Nazarbayev University, Astana, Kazakhstan; \\ ${ }^{b}$ Department of Electronic and Information Engineering, The Hong Kong Polytechnic University, \\ Hong Kong, P.R. China; ' Department of Physics, University of Hong Kong, Hong Kong, P.R. \\ China; ${ }^{\mathrm{d} D e p a r t m e n t}$ of Chemistry, University of Hong Kong, Hong Kong, P.R. China; ${ }^{\mathrm{e} S c h o o l}$ of \\ Engineering, Nazarbayev University, Astana, Kazakhstan.
}

\begin{abstract}
In this paper we report on systematic studies conducted for the improvement in both the device structure and the materials quality of perovskite based solar cells (PSCs). We have incorporated $\mathrm{TiO}_{2}$ nanorods, of length around 350-400 nm, in the device structure. Such structures were grown by solvothermal technique directly on the glass/FTO substrates. Characterization by femtosecond transient absorption (fs-TA) spectroscopy indicates that the incorporation of $\mathrm{TiO}_{2}-$ nanorod array (NA) greatly enhances the collection efficiency of the photo-generated carriers due to substantial reduction in carrier diffusion distance. To improve the crystallinity of the perovskite films we performed systematic studies on cryoassisted growth of the material. The technique eliminates the need for environmentally harmful anti-solvents and enables decoupling of the nucleation and crystallization phases by inhibiting chemical reactions in the precursor films rapidly cooled by immersion in liquid nitrogen. Furthermore, the technique leads to uniform precipitation of precursors due to the supersaturation condition in the residual solvents at cryogenic temperature resulting in highly uniform coverage of the films. Systematic characterization of the films by low-frequency noise and photothermal deflection technique indicate significant in the trap density of the films which is attributed as the main underlying reason for the observed improvement in the power conversion efficiency of the device. A high efficiency of $21.4 \%$ is achieved for our champion device.
\end{abstract}

Keywords: perovskite solar cells, cryo-assisted growth process, $\mathrm{TiO}_{2}$ nanorod arrays

\section{INTRODUCTION}

Modern society is characterized by an increasing demand for energy for the development of the infrastructure, growth of the economy and to raise the standard of living. The total energy consumption in 2010 is approximately 20 trillion $\mathrm{kWh}$ and is projected to reach 30 trillion $\mathrm{kWh}$ by 2030 . Securing clean and sustainable energy resources becomes a critical issue for the $21^{\text {st }}$ century or risk serious environmental consequences. Among the various renewable energy resources, solar energy is the most promising candidate for addressing the global energy challenge due to the abundance of solar energy that reaches the earth surface. On a cloudless day each square meter is receiving around $1 \mathrm{~kW}$ of power from the sun. Thus, simply covering $1 \%$ of the land area with photovoltaic cells is more than sufficient to satisfy the global energy needs. However, widespread use of solar power requires the development of cost-effective, high performance solar cells.

Recent emergence of the hybrid organometal halide perovskite as photovoltaic absorbers had led to important breakthroughs in the field of photovoltaics. The PSCs had accomplished dramatic improvement in the power conversion efficiency (PCE) from $3.8 \%$ in $2009^{[1]}$ to $23.7 \%$ as recently announced. ${ }^{[2]}$ Such superior performance is attributed to the high absorption coefficients, tunable bandgaps, long carrier diffusion length, high carrier mobility and low exciton binding energy of the perovskite material. ${ }^{[3]}$

Tremendous effort had been devoted for enhancing the performances of the devices through the optimization of the device architecture, deposition techniques as well as the compositions of different layers in the device. ${ }^{[4-6]}$ Development of high performance PSCs demands low defect density, high crystallinity, good coverage and uniformity ${ }^{[7-10]}$ Different deposition processes for perovskite thin films had been reported such as solution techniques, ${ }^{[1]]}$ thermal evaporation ${ }^{[12]}$ and a combination of vapor and the solution processes. ${ }^{[13-14]}$ Excellent coverage and uniformity for the perovskite films is a crucial factor in the production of high PCE devices. Among the different approaches, solvent engineering and the use of anti-solvent dripping was introduced, which facilitates better controlled reaction between the perovskite precursors due to the formation of a $\mathrm{CH}_{3} \mathrm{NH}_{3} \mathrm{I}-\mathrm{PbI}_{2}-\mathrm{DMSO}$ intermediary phase. ${ }^{[15]}$ The main function of the DMSO was to slow the

*charles.surya@nu.edu.kz 
reaction rate between $\mathrm{PbI}_{2}$ and $\mathrm{CH}_{3} \mathrm{NH}_{3} \mathrm{I}$ during spinning. The residual DMSO is removed by thermal annealing, resulting in perovskite films with high crystallinity and uniformity. The anti-solvent promotes rapid nucleation due to the initiation of an instantaneous local supersaturation of the precursor solution resulting in a uniform seed layer ${ }^{[15]}$ and is the most popular technique for the formation of high performance PSCs. However, commonly used anti-solvents such as chlorobenzene or toluene are environmentally harmful and highly toxic. Furthermore, there are important limitations in the scalability of technique as the perovskite film quality critically depends on the time, volume and position of the application of the anti-solvent as well as accurate control of the dripping process.

We introduce novel techniques for enhancing the performances of PSCs. We have investigated two different approaches. In the first approach we have introduced $\mathrm{TiO}_{2} \mathrm{NAs}$, grown by the solvothermal technique, in the perovskite absorber layer ${ }^{[16]}$ The introduction of NAs in the PSC has significant advantages for device performance, including a wide space for effective pore filling, a large surface area for carrier extraction, and a stabilized spatial distribution of straightforward nanochannels for electron transport. These factors will be beneficial for enhancing the extraction efficiency of photogenerated electrons and reducing the series resistance of the device. In addition, light scattering occurred amongst the NA ${ }^{[17]}$ is favorable to improving the photon absorption efficiency of the perovskite layer. Detailed investigations indicate significant enhancement in the collection efficiency of the photocarriers as well as the fill factor of the device.

The second strategy is to improve the crystallinity of the perovskite layer. We present a novel cryo-assisted growth process for perovskite thin films. The technique facilitates decoupling of the nucleation and crystallization processes and, hence, enables independent control of the two processes resulting in highly uniform nucleation sites for subsequent crystallization of perovskites. This is shown to be crucial for achieving high crystallinity in the perovskite films. Our method results in significant improvements in solar cell performance for different compositions of mixed perovskite films, indicating general applicability to different perovskite materials. ${ }^{[18]}$

\section{EXPERIMENT}

The patterned FTO on glass substrates were cleaned sequentially by ultrasonication in detergent solution followed by deionized (DI) water, acetone, and isopropanol. The substrates were then blow-dry by nitrogen gas which were then exposed to UV-ozone for 20 min before the deposition of the electron transport layer (ETL). The perovskite precursors used in this work included $\mathrm{PbI}_{2}, \mathrm{PbBr}_{2}, \mathrm{MAI}, \mathrm{MACl}, \mathrm{CsI}, \mathrm{MABr}$ and $\mathrm{FAI}$. The compositions of the perovskite were varied in order to optimize the film quality. The hole transport layer (HTL) was formed by spin-coating spiro-MeOTAD dissolved in chlorobenzene (CB) $(80 \mathrm{mg} / \mathrm{mL})$ with the additives of LiTFSI $(17.5 \mu \mathrm{L}$ from the early prepared stock solution of 520 $\mathrm{mg} / \mathrm{mL}$ in acetonitrile) and $29 \mu \mathrm{L}$ of tBP at $3000 \mathrm{rpm}$ for $30 \mathrm{~s}$. For metal electrode deposition, $80 \mathrm{~nm}-100 \mathrm{~nm}$ gold was thermally evaporated through a shadow mask defining the active area from $0.03 \mathrm{~cm}^{2}$ to $0.06 \mathrm{~cm}^{2}$. The details of the specific experimental conditions are disclosed in the section below.

\section{RESULTS AND DISCUSSION}

$\mathrm{TiO}_{2}$-NAs were grown on FTO coated glass by solvothermal technique in an autoclave using a precursor of $20 \mathrm{ml}$ of $\mathrm{H}_{2} \mathrm{O}, 20 \mathrm{ml}$ of $\mathrm{HCl}$ and $0.6 \mathrm{ml}$ of titanium isopropoxide. A typical SEM image of the NAs is shown in Fig. 1a which illustrates vertically aligned NAs on the FTO substrate with a length of $\sim 350-400 \mathrm{~nm}$, a diameter of $\sim 40-50 \mathrm{~nm}$ and rod density of $\sim 2 \times 10^{2} / \mu \mathrm{m}^{2}$. X-ray diffraction peaks for $\mathrm{TiO}_{2}$-NA match well with rutile $\mathrm{TiO}_{2}$ (JCPDS 86-0147), with a tetragonal crystal structure $\left(a=b=4.594 \AA, c=2.959 \AA\right.$, and $\left.\alpha=\beta=\gamma=90^{\circ}\right)$. The high-resolution STEM, as indicated in Fig. 1b, demonstrates that the $\mathrm{TiO}_{2}$-NA grown by the technique are single-crystalline with the interplanar distances of $d_{1}=0.295 \mathrm{~nm}$ and $d_{2}=0.324 \mathrm{~nm}$ which match well with the interplanar separations for the crystal planes along the (001) and (110) directions for rutile $\mathrm{TiO}_{2}$ respectively.

The main problem for the fabrication of NA-PSCs is the difficulty in the growth of high quality, continuous perovskite films on NA as the nanorods obstruct the flow of the precursor solution and, hence, make it difficult for the precursor solution to achieve uniform coverage over the substrate during the spin-coating process. Our strategy to grow high quality perovskite film on NAs starts with the synthesis of compact layer-free $\mathrm{TiO}_{2}-\mathrm{NA}$ at low temperature, the replacement of $\mathrm{MAI}-\mathrm{PbI}_{2}$ precursor system by the MACl- $\mathrm{PbI}_{2}$ system in DMSO and utilizing toluene dripping technique during the film formation process. We have investigated the morphology of the perovskite as a function of the MACl/MAI ratio. Five different ratios were used $(0,0.5,1,1.5$, and 2$)$ and the corresponding perovskite films (P0, P0.5, P1, P1.5 and P2) were characterized in detailed. Typical XRD patterns of the perovskite films are shown in Fig. 2. The FWHM values are $0.263^{\circ}$, $0.246^{\circ}, 0.223^{\circ}, 0.216^{\circ}$, and $0.215^{\circ}$ for the $\mathrm{P} 0, \mathrm{P} 0.5, \mathrm{P} 1, \mathrm{P} 1.5$, and $\mathrm{P} 2$ samples respectively which clearly indicate the improvement in the crystallinity with an increase in the $\mathrm{MACl}$ composition in the precursor. Among all conditions, the P1.5 sample exhibits the best crystallinity. 
a)

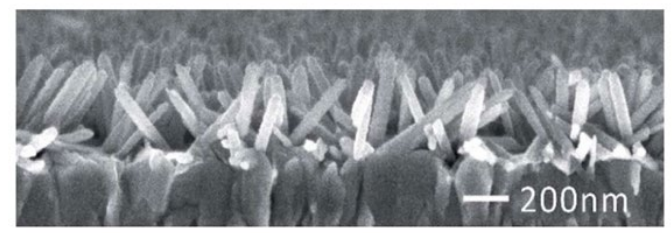

b)

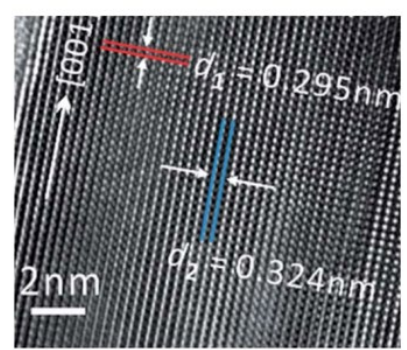

Fig. 1 a) The cross-sectional image of vertically aligned NAs on the FTO substrate; b) The high-resolution STEM image of $\mathrm{TiO}_{2}$-NA.

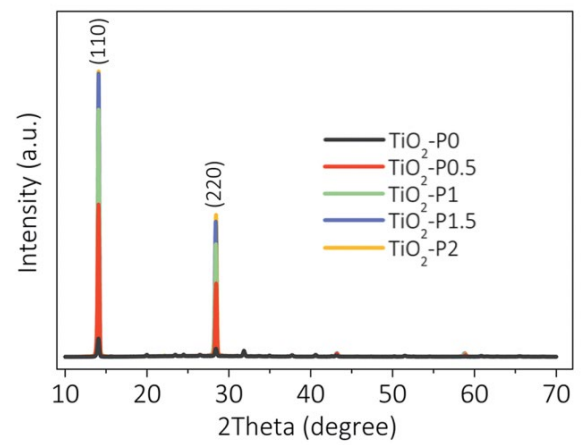

Fig. 2 The typical XRD patterns of the perovskite films with different MACl/MAI ratios.

Compared to $\mathrm{P} 0$ type sample, remarkable changes in the morphology can be seen when $\mathrm{MACl}$ is introduced into the precursor for perovskite growth, as shown in Fig. 3a-3e. The crystal sizes of the Cl-doped films have increased substantially from hundreds of nanometers to micrometer scale, indicating an enhanced crystallinity, which is consistent with the analyses from the XRD patterns. The density of the grain boundaries decreases significantly with the addition of MACl. From Fig. 3a-3c it can be seen that pinholes are still present in films with low MACl content (P0 - P1). By increasing the molar ratio of $\mathrm{MACl} / \mathrm{MAI}$ to 1.5 , the film exhibits full coverage and long-range crystallized morphology on $\mathrm{TiO}_{2}$-NA.
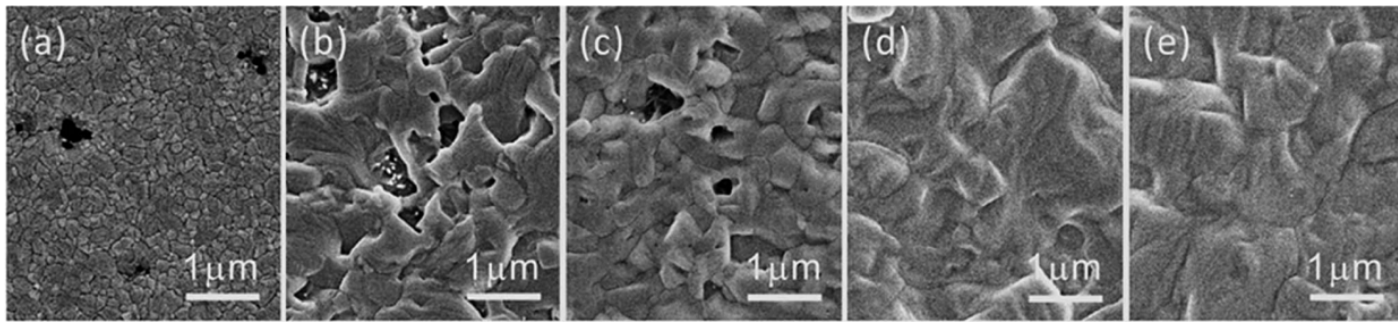

Fig. 3 The morphology of perovskite films with different MACl/MAI ratios a) 0; b) 0.5 ; c) 1 ; d) 1.5 and e) 2 .

Detailed characterizations of the photothermal deflection spectroscopy (PDS) were performed to obtain insights into the quality of the perovskite films grown with different $\mathrm{MACl}$ concentration. The PDS technique can be used to detect the 
energetic disorders as the exponential decay of the sub-bandgap absorption as characterized by the Urbach energy (Eu). ${ }^{[12]}$ The Urbach energy reflects the steepness of the band tail states at the conduction band edge. A smaller Eu stands for a lower density of shallow trap states. ${ }^{[12]}$ From Fig. 4a the Eu values are $23.2 \mathrm{meV}, 20.9 \mathrm{meV}, 20.7 \mathrm{meV}, 20.5 \mathrm{meV}$, and $20.8 \mathrm{meV}$ for the P0, P0.5, P1, P1.5, and P2 films respectively. Thus, the introduction of MACl is favorable to morphology improvement as well as suppressing the shallow trap states. The data show Eu reaches a minimum for the P1.5 film Indicating that P1.5 film has the lowest degree of energetic disorders and excessive MACl would introduce again some shallow trap states to the perovskite film. From the PDS spectra it is observed that a reduction in deep trap states for the $\mathrm{P} 0.5$ to $\mathrm{P} 2$ films compared to the P0 film particularly in the range of 0.6 to $1.1 \mathrm{eV}$ and $\mathrm{P} 1.5$ is found to exhibit the lowest level. Further increase in $\mathrm{MACl}$ content (i.e. P2) leads to enhanced absorption, suggesting an increase in the deep trap states. Using P1.5 type films for the fabrication of PSC, a high PCE of $>19 \%$ and a fill factor of $\sim 78 \%$ is achieved under AM $1.5 \mathrm{G}\left(100 \mathrm{~mW} / \mathrm{cm}^{2}\right)$, has been achieved (Fig. $4 \mathrm{~b}$ ), demonstrating the great potential of $\mathrm{TiO}_{2}-\mathrm{NA}$ as the ETL compared to their planar and mesoporous counterparts for application in PSCs.

a)

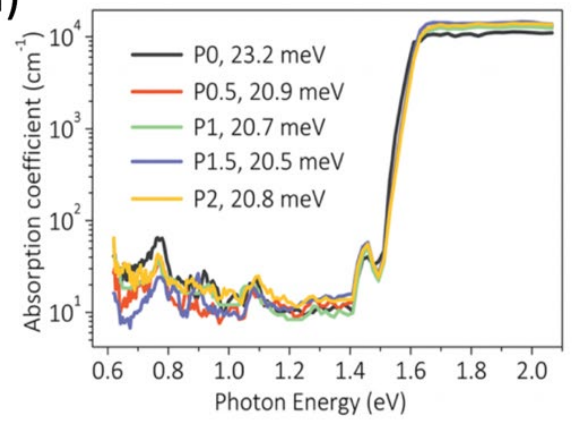

b)

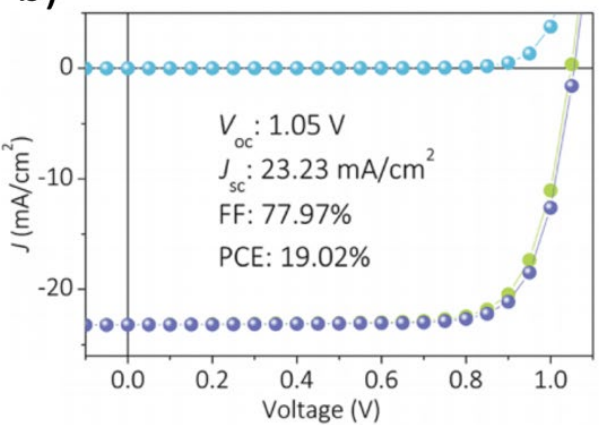

Fig. 4 a) The PDS spectra of the perovskite films with different MACl/MAI ratios; b) The I-V curves of the NA-PSC with type P1.5 perovskite films.

Femtosecond time-resolved spectroscopy (fs-TA) experiments were performed on the P1.5 perovskite films grown on a $\mathrm{TiO}_{2}$ compact layer (CL-P1.5) as a control experiment as well as the NA substrate. By means of such ultrafast transient absorption measurements, one can obtain the extraction times at the interfaces between the perovskite and a typical $\mathrm{TiO}_{2}-$ $\mathrm{NA}$ or the $\mathrm{TiO}_{2}$ compact layer. Figures $5 \mathrm{a}$ and $5 \mathrm{~b}$ exhibit the fs-TA spectra for the control and the P1.5-NA samples. The control sample exhibits a little stronger fluorescence, indicating that the $\mathrm{TiO}_{2}$ compact layer is less effective than $\mathrm{TiO}_{2}$ NA to quench the photoluminescence of the perovskite layer. This is attributed to the larger interfacial area between the $\mathrm{P} 1.5$ perovskite and $\mathrm{TiO}_{2}-\mathrm{NA}$ as well as the penetration of the $\mathrm{TiO}_{2}-\mathrm{NA}$ deep into perovskite layer and thereby significantly enhancing the collection of the photo-carriers through the reduction of their diffusion distance. This model is corroborated by the longer extraction time of the type CL-P1.5 junction ( $\tau=436 \mathrm{ps})$ compared to the P1.5-NA ( $\tau=317 \mathrm{ps})$ junction.

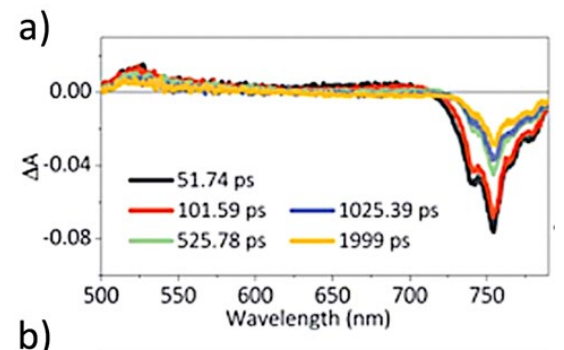

b)

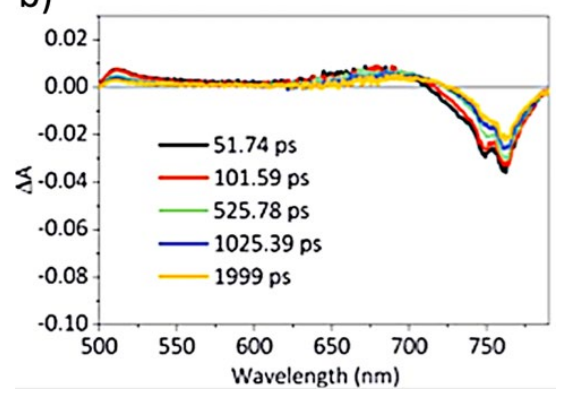

Fig. 5 The spectra obtained from the fs-TA measurement for perovskite films deposited on a) compact layer (CL$\mathrm{P} 1.5)$ and b) $\mathrm{TiO}_{2}$-NA (P1.5 NA) 
The second strategy is to enhance the performance of PSCs is achieved through the improvement in the crystallinity of the perovskite layer. We have developed a novel cryo-controlled nucleation technique, which effectively decouples the nucleation and crystallization phases and ensures the formation of a uniform seed layer for subsequent perovskite growth. The schematic diagrams shown in Fig. 6 illustrate the process for the cryo-assisted technique. First, the precursors were spin-coated onto a glass/FTO/ $\mathrm{SnO}_{2}$ substrate. This is followed by dipping the spin-coated film into a liquid nitrogen $\left(\mathrm{LN}_{2}\right)$ bath immediately. The rapid reduction in temperature induced abrupt solidification of the solvent, consisting of a mixture of DMF (m.p. $-61^{\circ} \mathrm{C}$ ) and DMSO (m.p. $19^{\circ} \mathrm{C}$ ), which prevented chemical reaction and ensured the uniform distribution of the solutes. The chemical reaction rate was basically reduced to zero due to the low temperature and effectively prevents formation of large perovskite crystallites prematurely. Upon removal from the $\mathrm{LN}_{2}$, the temperature of the substrate increased slowly to above the melting point of the solvent $\left(\sim-50^{\circ} \mathrm{C}\right)$. This is followed by blow drying the sample using dry $\mathrm{N}_{2}$ to remove the residual solvents in the precursor film. The technique leads to uniform precipitation of the precursors over the entire substrate due to the supersaturated condition induced in the solution. Finally, the sample was heated to convert the precursors into the perovskite material.

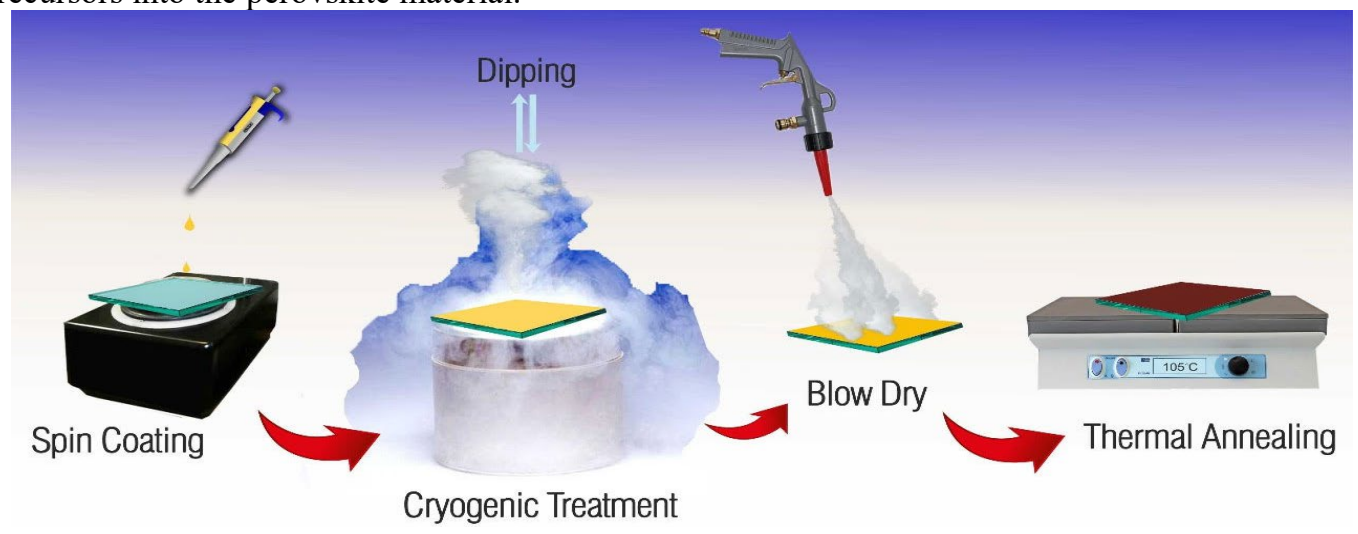

Fig. 6 The schematic diagrams illustrate the processes for the cryo-assisted technique for growing the perovskite film.

Using this process, we were able to obtain very smooth perovskite films with excellent coverage over the entire substrate as shown in Fig. 7b, which is very different from the films deposited using the conventional method as indicated in the insets of Fig. 7a.

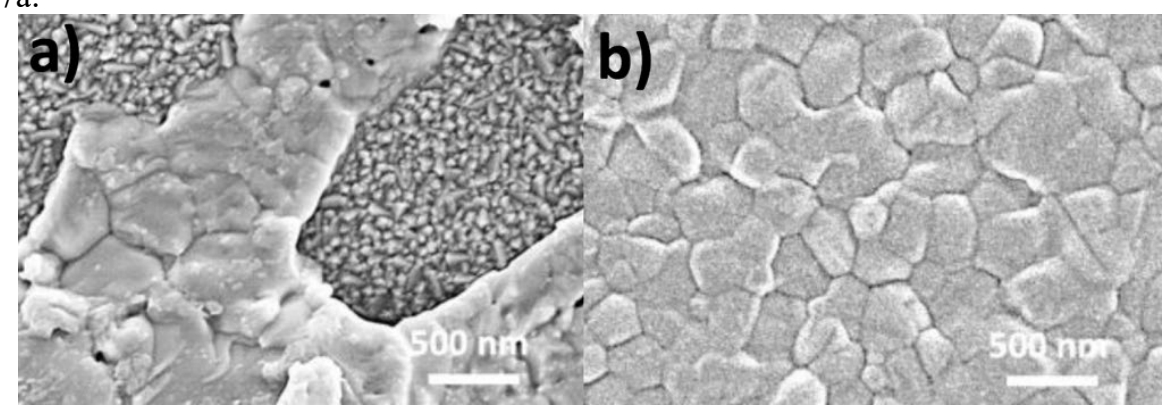

Fig. 7 The morphology of mixed perovskite films $\left(\mathrm{Cs}_{0.05}\left(\mathrm{MA}_{0.17} \mathrm{FA}_{0.83}\right)_{0.95} \mathrm{~Pb}\left(\mathrm{I}_{0.84} \mathrm{Br}_{0.16}\right)_{3}: \mathrm{Cl}\right)$ prepared by the a) conventional method and b) cryo-assisted technique.

X-ray diffraction characterizations on $\mathrm{Cs}_{0.05}\left(\mathrm{MA}_{0.17} \mathrm{FA}_{0.83}\right)_{0.95} \mathrm{~Pb}\left(\mathrm{I}_{0.84} \mathrm{Br}_{0.16}\right)_{3}: \mathrm{Cl}$ films provide further insight into the evolution of mixed perovskite films growth with (line 2) and without (line 1) cryogenic treatment. The characteristic XRD peaks are dominant at $14.1^{\circ}$ and $28.4^{\circ}$ for both lines 1 and 2 in Fig. 8. However, the crystallinity of the control film is still substantially lower compared to the sample with cryogenic treatment. 


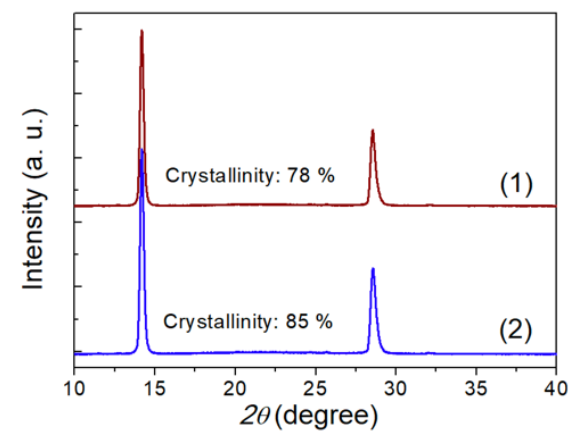

Fig. 8 The X-ray diffraction patterns for prepared perovskite films with (line 2) or without (line 1) cryogenic treatment.

We performed low-frequency noise (LFN) measurements on both the control and $\mathrm{Cs}_{0.05}\left(\mathrm{MA}_{0.17} \mathrm{FA}_{0.83}\right)_{0.95}$ $\mathrm{Pb}\left(\mathrm{I}_{0.84} \mathrm{Br}_{0.16}\right)_{3}: \mathrm{Cl}$ samples growth with and without using cryogenic treatment. The noise power spectral density (PSD) is expressed in a functional form of $S(f) \propto 1 / f^{\gamma}$ where $\gamma \approx 1$. It has been shown that LFN originates from the random trapping and detrapping of carriers by localized states in the material ${ }^{[19]}$ resulting in the corresponding fluctuation in the conductance of the device in the form of a random telegraph noise with the noise PSD given by

$$
S(f)=\frac{A \tau}{1+4 \pi^{2} f^{2} \tau^{2}}
$$

where $A$ is a proportionality constant and $\tau$ is the fluctuation time constant. It has been showed that LFN of PSCs arises from a thermally activated trapping and detrapping process, ${ }^{[8]}$ hence the fluctuation time constant is given by $\tau=\tau_{0} \exp \left(\frac{E}{k_{B} T}\right)$, where $\tau_{0}$ is of the order of the inverse phonon frequency of the order $10^{-12} \mathrm{~s}, E$ is the activation energy of the trap, $k_{B}$ is the Boltzmann constant and $T$ is the absolute temperature. Since the individual trapping and detrapping events due to different trap states are statistically independent, the overall current noise PSD, $S_{I}(f)$, for a large device with large number of localized states can be expressed as

$$
\frac{S_{I}(f)}{I^{2}}=A \int_{x} \int_{y} \int_{z} \int_{E} N_{T}(E, x, y, z) \frac{\tau}{1+4 \pi^{2} f^{2} \tau^{2}} d E d z d y d x,
$$

where $N_{T}$ is the trap density and $I$ is the dc current applied to the device. Based on the thermal activation model for the LFN, the trap density $N_{T}$ can be expressed as

$$
N_{T}\left(E_{p}\right) \approx \frac{4 C f}{k_{B} T} \frac{S_{I}(f)}{I^{2}}=\frac{4 C f}{k_{B} T} \frac{S_{V}(f)}{V^{2}},
$$

where $C$ is a proportionality constant and the Lorentzian peaks sharply at $E_{p}=-k_{B} T \ln \left(\omega \tau_{0}\right)$. To pinpoint the impact of material growth technique on the defect density of perovskites, we have performed the LFN measurement on the samples with a resistive structure (FTO/perovskite/Au), which not only characterizes the bulk of the perovskite layer but also the perovskite/FTO and perovskite/Au interfaces as well. Based on Eq. 3 above the normalized trap densities, $N_{T N}(E)$ were computed for the samples under test by adopting a unity value for $C$ and the results are shown in Fig. 9a. The results demonstrate substantial decrease in the trap density for the $\mathrm{Cs}_{0.05}\left(\mathrm{MA}_{0.17} \mathrm{FA}_{0.83}\right)_{0.95} \mathrm{~Pb}\left(\mathrm{I}_{0.84} \mathrm{Br}_{0.16}\right)_{3}: \mathrm{Cl}$ sample prepared by the 4-step growth technique compared to the control. A champion PCE of $21.4 \%$ with a $V_{O C}=1.14 \mathrm{~V}, J_{S C}=23.5 \mathrm{~mA} / \mathrm{cm}^{2}$ and $\mathrm{FF}=0.80$ can be achieved for PSC by using the proposed cryo-assisted perovskite growth method (Fig. $9 \mathrm{~b}$ ).

a)

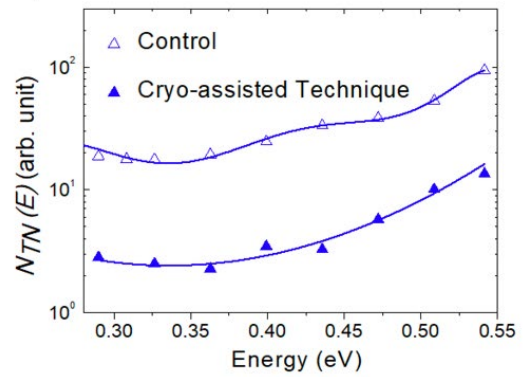

b)

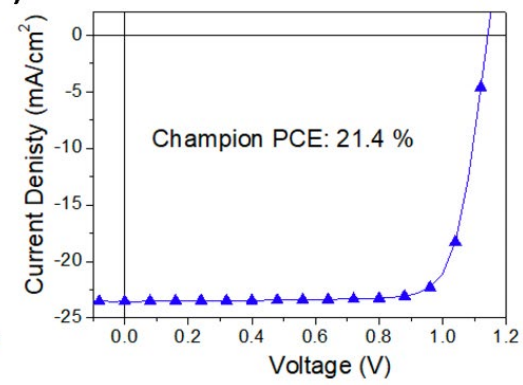

Fig. 9 a) The results of LFN measurement on the samples in a resistive structure (FTO/perovskite (with or without cryogenic treatment cryo-assisted technique)/Au); b) The $I-V$ curve of the champion PSC prepared by the cryo-assisted perovskite growth method. 


\section{CONCLUSIONS}

We have investigated the incorporation of $\mathrm{TiO}_{2}$-NAs in PSCs. It is shown that the deposition parameters need to be adjusted to ensure the growth of high-quality perovskite thin films on NAs. We have optimized MACl/MAI ratio together with the addition of DMSO in the precursor solution and applying anti-solvent dripping method during the spin-coating process to ensure the growth a uniform perovskite film with high crystallinity. We have also demonstrated that, with proper deposition process, the incorporation of $\mathrm{TiO}_{2}-\mathrm{NA}$ in the PSCs can greatly facilitate the collection of photo-generated carriers in the devices due to the larger interfacial area between the perovskite and the $\mathrm{TiO}_{2}$-NA. By using the proposed techniques, a PCE of $19.02 \%$ with negligible hysteresis can be achieved for the device without the high temperature processing $\mathrm{TiO}_{2} \mathrm{CL}$, which is a record performance for the NAPSCs. This demonstrates the superiority of $\mathrm{TiO}_{2}$-NA as an ETL compared to their planar and mesoporous counterparts for applications in PSCs.

A novel 4-step cryo-controlled method for the deposition of high-quality mixed perovskite films without the use of anti-solvents has been demonstrated. This method results in increased grain size and crystallinity and lower defect density for the mixed perovskite films under study. Processing at cryogenic temperature suppresses premature reactions of the precursors and prevents premature coalescence of nuclei into large crystallites, enabling uniform film formation following the blow-drying and annealing steps. The method is of particular interest for Cs-containing perovskite formulations, since it is shown to successfully suppress the formation of the undesirable yellow phase $\delta$ - $\mathrm{CsPbI}_{3}$. Using this method, a champion PCE of $21.4 \%$ with a $V_{O C}=1.14 \mathrm{~V}, J_{S C}=$ $23.5 \mathrm{~mA} / \mathrm{cm}^{2}$ and $\mathrm{FF}=0.80$ can be achieved for optimized perovskite composition.

\section{ACKNOWLEDGEMENTS}

This work was supported by Nazarbayev University Grant Award No. 090118FD5326 and the targeted Program BR05236524. Special thanks are given to Prof. S. K. So and Mr. S. H. Cheung for professional PDS characterizations.

\section{REFERENCES}

[1] Kojima, A., Teshima, K., Shirai, Y. and Miyasaka, T., "Organometal halide perovskites as visible-light sensitizers for photovoltaic cells,” J. Am. Chem. Soc. 131(17), 6050-6051 (2009).

[2] National Renewable Energy Laboratory, Best research-cell efficiencies, https://www.nrel.gov/pv/assets/pdfs/pvefficiency-chart.20190103.pdf (accessed: Jan 2019).

[3] Ansari, M. I. H., Qurashi, A. and Nazeeruddinc, M. K., "Frontiers, opportunities, and challenges in perovskite solar cells:A critical review," J. Photochem. Photobiol., C 35, 1-24 (2018).

[4] Dong, Q., Liu, F., Wong, M. K., Tam, H. W., Djurišić, A. B., Ng, A., Surya, C., Chan, W. K. and Ng, A. M. C., “ Encapsulation of perovskite solar cells for high humidity conditions," ChemSusChem 9 (18), 2597-2603 (2016).

[5] Chen, W., Wu, Y., Fan, J., Djurišić, A. B., Liu, F., Tam, H. W., Ng, A., Surya, C., Chan, W. K., Wang, D. and He, Z.-B., "Understanding the doping effect on NiO: toward high - performance inverted perovskite solar cells," Adv. Energy Mater. 8 (19), 1703519 (2018).

[6] Djurišić, A. B., Liu, F. Z., Tam, H. W., Wong, M. K., Ng, A., Surya, C., Chen, W. and He, Z. B., "Perovskite solar cells - An overview of critical issues," Prog. Quantum Electron. 53, 1-37 (2017).

[7] Djurišić, A. B., Liu, F. Ng, A. M. C., Dong, Q., Wong, M. K., Ng, A. and Surya, C., "Stability issues of the next generation solar cells," Phys. Status Solidi RRL 10 (4), 281-299 (2016).

[8] Shen, Q., Ng, A., Ren, Z., Gokkaya, H. C., Djurišić, A. B., Zapien, J. A. and Surya, C., "Characterization of lowfrequency excess noise in $\mathrm{CH}_{3} \mathrm{NH}_{3} \mathrm{PbI}_{3}$-based solar cells grown by solution and hybrid chemical vapor deposition techniques" ACS Appl. Mater. Interfaces 10 (1), 371-380 (2018)

[9] Liu, F., Dong, Q. Wong, M. K., Djurišić, A. B., Ng, A. Ren, Z., Shen, Q., Surya, C., Chan, W. K., Wang, J., Ng, A. M. C., Liao, C. and Shih, H. Li, K., "Is excess $\mathrm{PbI}_{2}$ beneficial for perovskite solar cell performance?," Adv. Energy Mater. 6 (7), 1502206 (2016).

[10] Ren, Z., Ng, A., Shen, Q., Gokkaya, H. C., Wang, J., Yang, L., Yiu, W.-K., Bai, G., Djurišić, A. B., Leung, W. W., Hao, J., Chan, W. K. and Surya, C., "Thermal assisted oxygen annealing for high efficiency planar $\mathrm{CH}_{3} \mathrm{NH}_{3} \mathrm{PbI}_{3}$ perovskite solar cells," Sci. Rep. 4, 6752 (2014). 
[11] Das, J., Subbiah, A. S., Mahuli, N., Singh, R. and Sarkar, S. K., "One-step solution-processed formamidinium lead tribromide formation for better reproducible planar perovskite solar cells" Energy Technol. 5 (10), 1807-1813 (2107). [12] Ng, A., Ren, Z., Shen, Q., Cheung, S. H., Gokkaya, H. C., Bai, G., Wang, J., Yang, L., So, S. K., Djurišić, A. B., Leung, W. W., Hao, J., Chan, W. K. and Surya, C. "Efficiency enhancement by defect engineering in perovskite photovoltaic cells prepared using evaporated $\mathrm{PbI}_{2} / \mathrm{CH}_{3} \mathrm{NH}_{3} \mathrm{I}$ multilayers " J. Mater. Chem. A 3 (17), 9223-9231 (2015). [13] Wong, M. K., Liu, F., Kam, C. S., Leung, T. L., Tam, H. W., Djurišić, A. B., Popović, J., Li, H., Shih, K., Low, K.H., Chan, W. K., Chen, W., He, Z., Ng, A. and Surya, C., "Synthesis of lead-free perovskite films by combinatorial evaporation: fast processes for screening different precursor combinations," Chem. Mater. 2017, 29 (23), 9946-9953. [14] Ng, A., Ren, Z., Shen, Q., Cheung, S. H., Gokkaya, H. C., So, S. K., Djurišić, A. B., Wan, Y., Wu, X., and Surya, C., "Crystal engineering for low defect density and high efficiency hybrid chemical vapor deposition grown perovskite solar cells," ACS Appl. Mater. Interfaces 8(48), 32805-32814 (2016).

[15] Jeon, N. J., Noh, J. H., Kim, Y. C., Yang, W. S., Ryu, S. and Seok, S. I., "Solvent engineering for high-performance inorganic-organic hybrid perovskite solar cells," Nat. Mater. 13 (9), 897-903 (2014).

[16] Liu, C., Zhu, R., Ng, A., Ren, Z., Cheung, S. H., Du, L., So, S. K., Zapien, J. A., Djurišić, A. B. and Phillips, D. L., Surya, C., "Investigation of high performance $\mathrm{TiO}_{2}$ nanorod array perovskite solar cells" J. Mater. Chem. A 5 (30), 15970-15980 (2017).

[17] Muskens, O. L., Rivas, J. G., Algra, R. E., Bakkers, E. P. A. M. and Lagendijk, A., "Design of light scattering in nanowire materials for photovoltaic applications," Nano Lett., 8 (9), 2638-2642 (2008).

[18] Ng, A., Ren, Z., Hu, H., Fong, P. W. K., Shen, Q., Cheung, S. H., Qin, P., Lee, J.-W., Djurišić, A. B., So, S. K., Li, G., Yang, Y. and Surya, C., "A cryogenic process for antisolvent-free high-performance perovskite solar cells," Adv. Mater. 30(44), 1804402 (2018).

[19] Vandanne, L. K. J., Alabedra, R., and Zonniti, M., "1/f noise as a reliability estimation for solar cells", Solid State Electron. 26 (7), 671-674 (1983). 